\title{
BOUNDS FOR THE CHARACTERISTIC ROOTS OF A MATRIX
}

\author{
EDWARD W. BARANKIN
}

In a recent paper $[1],{ }^{1} \mathrm{~A}$. B. Farnell reiterated the possibility of the existence of a certain upper bound for the characteristic roots of a matrix. This bound was first conjectured by W. V. Parker (cf. [1]), and was, in fact, proved in the case of matrices of order 2 by Farnell. We shall here, in Theorem 1 below, establish that bound for matrices of any order $n$. Two previous results on bounds for characteristic roots, one due to Parker [2], and one to Farnell [1], will be stated for purposes of comparison with Theorem 1. But first, we make certain definitions, following, for the most part, those of Farnell, for the sake of uniformity.

We let $A=\left\|a_{r s}\right\|$, of order $n$, be any matrix of complex numbers, with a characteristic $\operatorname{root} \lambda=\alpha+i \beta$ different from 0 . Then there exists a non-null vector $\left\{x_{r}\right\}, r=1, \cdots, n$, such that

$$
\lambda x_{r}=\sum_{s} a_{r s} x_{s} .
$$

With $A^{*}$ denoting the hermitian-conjugate of $A$, set

$$
\left(A+A^{*}\right) / 2=B=\left\|b_{r s}\right\|, \quad\left(A-A^{*}\right) / 2 i=C=\left\|c_{r s}\right\|,
$$

both of which matrices are hermitian. Finally, define

$$
\begin{aligned}
R_{r}=\sum_{s}\left|a_{r s}\right|, & T_{s}=\sum_{r}\left|a_{r s}\right|, \\
R=\max _{(r)} R_{r}, & T=\max _{(s)} T_{s}, \\
S_{r}^{\prime}=\sum_{s^{\prime}}\left|b_{r s}\right|=\sum_{s}\left|b_{s r}\right|, & S_{r}^{\prime}=\sum_{s}\left|c_{r s}\right|=\sum_{s}\left|c_{s r}\right| .
\end{aligned}
$$

Now the theorems of Parker and Farnell may be stated briefly as follows :

PARKer's TheOREM. If $S, S^{\prime}$, and $S^{\prime \prime}$ are the greatest of the $\left(R_{r}+T_{r}\right) / 2, S_{r}^{\prime}$, and $S_{r}^{\prime \prime}$, respectively, then

$$
|\lambda| \leqq S, \quad|\alpha| \leqq S^{\prime}, \quad|\beta| \leqq S^{\prime \prime} .
$$

FARNELL'S TheOREM. ${ }^{2}|\lambda|^{2}$ is bounded above by $R T$.

Presented to the Society, February 24, 1945; received by the editors March 20, 1945.

${ }^{1}$ Numbers in brackets refer to the references cited at the end of the paper.

${ }^{2}$ This is not the strongest bound that Farnell obtains. 
It should be pointed out that both these theorems were proved for any $\lambda$ in the field of values of the matrix $A$, and thereby in particular for the characteristic roots. On the other hand, the results that we shall establish below are not necessarily true for all numbers in the field of values. One observes that these theorems, and all that follow, are trivially true when $\lambda=0$. We therefore assume throughout that $\lambda \neq 0$.

We now prove the following theorem.

THEOREM 1. $|\lambda|^{2}$ is bounded above by $\max _{(r)}\left(R_{r} T_{r}\right)$.

From the equalities (1) we deduce immediately the inequalities

$$
|\lambda|\left|x_{r}\right| \leqq \sum_{s}\left|a_{r s}\right|\left|x_{s}\right|=\sum_{s}\left|a_{r s}\right|^{1 / 2} \cdot\left|a_{r s}\right|^{1 / 2}\left|x_{s}\right| .
$$

Applying Schwarz's inequality to the right-hand side in the manner indicated by the preparation, we obtain

or, squaring this,

$$
\begin{aligned}
|\lambda|\left|x_{r}\right| & \leqq\left\{\left(\sum_{s}\left|a_{r s}\right|\right)\left(\sum_{s}\left|a_{r s}\right|\left|x_{s}\right|^{2}\right)\right\}^{1 / 2} \\
& =R_{r}^{1 / 2}\left(\sum_{s}\left|a_{r s}\right|\left|x_{s}\right|^{2}\right)^{1 / 2}
\end{aligned}
$$

$$
|\lambda|^{2}\left|x_{r}\right|^{2} \leqq R_{r} \sum_{s}\left|a_{r s}\right|\left|x_{s}\right|^{2}
$$

Suppose first that no $R_{r}$ vanishes. Then we divide the $r$ th inequality by $R_{r}$, and sum over $r$, so obtaining

$$
|\lambda|^{2} \sum_{r} \frac{\left|x_{r}\right|^{2}}{R_{r}} \leqq \sum_{r, s}\left|a_{r s}\right|\left|x_{s}\right|^{2}=\sum_{s} T_{s}\left|x_{s}\right|^{2}
$$

This may be written

$$
\sum_{r}\left(\frac{|\lambda|^{2}}{R_{r}}-T_{r}\right)\left|x_{r}\right|^{2} \leqq 0 .
$$

It follows that, for some $m$,

or

$$
\frac{|\lambda|^{2}}{R_{m}}-T_{m} \leqq 0
$$

$$
\left|\lambda^{2}\right| \leqq R_{m} T_{m} \leqq \max _{(r)}\left(R_{r} T_{r}\right),
$$

and the theorem is proved if $R_{r} \neq 0$ for each $r$. 
Now suppose that some $R_{r}$ vanishes. By a permutation of the inequalities (3), and a like permutation of the terms in the right-hand member of each inequality (a similarity transformation of the matrix $A !)$, we may take $R_{n}=0$. Then, since $\lambda \neq 0$, we must have $x_{n}=0$. The inequalities (3), which are $n$ in number, and in each of which the summation extends over the range $s=1,2, \cdots, n$, are thus reduced to the $n-1$ inequalities

$$
|\lambda|^{2}\left|x_{r}\right|^{2} \leqq R_{r} \sum_{s=1}^{n-1}\left|a_{r s}\right|\left|x_{s}\right|^{2}, \quad r=1,2, \cdots, n-1 .
$$

If, now, $R_{r} \neq 0$ for $r=1,2, \cdots, n-1$, we apply to these inequalities the arguments above, and obtain

$$
|\lambda|^{2} \leqq \max _{(r)}\left(R_{r} \cdot \sum_{s=1}^{n-1}\left|a_{s r}\right|\right) .
$$

But $R_{n}=0$ implies that $\left|a_{n r}\right|=0$ for each $r=1,2, \cdots, n$. Hence, for each $r$,

$$
\sum_{s=1}^{n-1}\left|a_{s r}\right|=\sum_{s=1}^{n}\left|a_{s r}\right|=T_{r}
$$

and so, again, as was to be proved,

$$
|\lambda|^{2} \leqq \max _{(r)}\left(R_{r} T_{r}\right) \text {. }
$$

In general, by the procedure just illustrated, one shows that

$$
|\lambda|^{2} \leqq \max _{(r)}\left(R_{r} \cdot \sum_{\tau}\left|a_{\tau r}\right|\right)
$$

where $\tau$ ranges over those indices $\kappa$ such that $R_{\kappa} \neq 0$. But for those indices $s$, such that $R_{s}=0$, one has $\left|a_{s r}\right|=0$ for each $r=1,2, \cdots, n$, so that

$$
\sum_{r}\left|a_{r r}\right|=\sum_{s=1}^{n}\left|a_{s r}\right|=T_{r}
$$

Thus, the theorem is proved in every case.

Parker's bound on $|\lambda|$ follows from Theorem 1 , since if $|\lambda|^{2} \leqq R_{m} T_{m}$, then

$$
|\lambda| \leqq\left(R_{m} T_{m}\right)^{1 / 2} \leqq\left(R_{m}+T_{m}\right) / 2 \leqq S .
$$

Furthermore, if $|\lambda|^{2} \leqq R_{m} T_{m}$, then $R_{m} \leqq R$ and $T_{m} \leqq T$, and we have $|\lambda|^{2} \leqq R T$, which is Farnell's result.

By the method employed above, one can establish the following, somewhat stronger theorem. 
Theorem 2. Let $0 \leqq k \leqq 2$, and define $R_{r}^{(k)}=\sum_{s}\left|a_{r s}\right|^{k}, T_{s}^{(k)}=\sum_{r}\left|a_{r s}\right|^{k}$. Then, for each fixed $k$ in the specified interval, $|\lambda|{ }^{2}$ is bounded above by $\max _{(r)}\left(R_{r}^{(k)} T_{r}^{(2-k)}\right)$.

To prove this, one applies the very same train of arguments as in the proof of Theorem 1, starting with the inequalities (2) in the form

$$
|\lambda|\left|x_{r}\right| \leqq \sum_{s}\left|a_{r s}\right|^{k / 2} \cdot\left|a_{r s}\right|^{1-k / 2}\left|x_{s}\right| \text {. }
$$

By permitting $k$ to vary with $r$ and $s$ in these last inequalities, one obtains a still stronger generalization of Theorem 1 .

We state one more result, which obtains without the aid of Schwarz's inequality, and which again implies Farnell's theorem.

Theorem 3. $|\lambda|$ is bounded above by both $R$ and $T$.

Let $\left|x_{m}\right|=\max _{(r)}\left|x_{r}\right|$; then, dividing the inequality

$$
|\lambda|\left|x_{m}\right| \leqq \sum_{s}\left|a_{m s}\right|\left|x_{s}\right|
$$

through by $\left|x_{m}\right|$, we have

$$
|\lambda| \leqq \sum_{s}\left|a_{m s}\right| \frac{\left|x_{s}\right|}{\left|x_{m}\right|} \leqq \sum_{s}\left|a_{m s}\right|=R_{m} \leqq[R .
$$

Now, sum all the following inequalities:

we obtain

$$
|\lambda|\left|x_{r}\right| \leqq \sum_{s}\left|a_{r s}\right|\left|x_{s}\right|
$$

or

$$
|\lambda| \sum_{r}\left|x_{r}\right| \leqq \sum_{s} T_{s}\left|x_{8}\right|
$$

$$
\sum_{s}\left(|\lambda|-T_{s}\right)\left|x_{8}\right| \leqq 0 \text {. }
$$

Hence, for some $m$,

$$
\begin{gathered}
|\lambda| \leqq T_{m} \leqq T . \\
\text { REFERENCES }
\end{gathered}
$$

1. Farnell, Limits for the characteristic roots of a matrix, Bull. Amer. Math. Soc. vol. 50 (1944) pp. 789-794.

2. Parker, The characteristic roots of a matrix, Duke Math. J. vol. 3 (1937) pp. 484-487.

University of California 